\title{
The role of Aotearoa New Zealand midwives as positive influencers on food literacy with Samoan families: Report on a small Auckland-based study
}

\author{
Hoy Neng Wong Soon ${ }^{A, B}$ MHSC, BA, GradDip, CA • Ineke Crezee ${ }^{B}$ PhD, MA, BA, RGON • Elaine
} Rush $^{B}$ PhD, MSC

${ }^{\text {A }}$ Corresponding author: hoyneng. wongsoon@aut.ac.nz

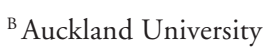
of Technology,

Auckland, Aotearoa New Zealand

\begin{abstract}
Background: Healthy eating is crucial for optimal development during all stages of life and most particularly during pregnancy. According to Stats NZ, Pasefika people make up $8.1 \%$ of the total Aotearoa New Zealand population. Information from the Ministry of Health suggests that Pasefika people have the highest level of food insecurity and the highest level of obesity. Women are more likely than men to be involved with food preparation; therefore, it is important to know to what extent women are aware of what healthy eating means for themselves and their families.
\end{abstract}

Aim: This study aimed to explore an understanding of the levels of food literacy among representatives of three generations of women in five Samoan families; how each of the three generations ensured that their dietary intake contributed to their quality of health; and whether the extent of food literacy was influenced both within and between/across generations.

Method: The study used a combined Delphi-Talanoa approach to interview 15 Samoan women about the levels of their understanding of food literacy.

Findings: Midwives played an important role in helping pregnant Samoan women understand about healthy eating during pregnancy. Women shared with other female relatives what they had learned about healthy eating from their midwives. Learnings taken from midwives included the importance of choosing healthy foods, portion control and physical activity.

Conclusion: All study participants who had received antenatal care in Aotearoa New Zealand mentioned the important role of midwives in increasing their understanding of healthy lifestyle choices. What the women learned from their midwives also informed their wider family or aiga.

Keywords: dietary intake, food, gestational diabetes, healthy conversations, lifestyle choices, midwives, positive influencers

\section{INTRODUCTION}

This article discusses some of the findings of a study undertaken in the context of a Master of Health Science thesis which examined the food literacy of a group of Samoan women belonging to three generations within five families. In general, women are more likely than men to be involved in the planning and preparation of meals and selection of food (Barreiro-Hurlé et al., 2010). It is therefore important to know to what extent women are aware of how to eat well for their own and their children's health (cf. Machín et al., 2016). A number of participants in the original study mentioned the role of midwives as positive influencers in terms of what women ate and how much they moved.

Healthy eating is crucial for optimal development during all stages of life and most particularly during pregnancy. According to Stats NZ (2019), Pasefika people make up $8.1 \%$ of the total population of Aotearoa New Zealand (Aotearoa NZ). Information from the Ministry of Health (MOH, 2019a, 2019b) suggests that
Pasefika people living in Aotearoa NZ have the highest level of food insecurity and the highest level of obesity. Women are more likely than men to be involved with food preparation; therefore, it is important to know to what extent women are aware of what healthy eating means for themselves and their families. The study this article is based on aimed to explore an understanding of the levels of food literacy among representatives of three generations of women in five Samoan families. It aimed to find out how each of the three generations ensured that their dietary intake contributed to their quality of health; and whether the degree of knowledge about food literacy was influenced both within and between/across generations. The study used a combined DelphiTalanoa approach to interview 15 Samoan women about their understanding of what are healthy and unhealthy foods, in order to gauge the level of food literacy and how it varies from generation to generation and between families, within a specified ethnic group. The original study did not set out to explore the role of midwives, 
but interviews reflected the important role the latter had played as positive influencers on food literacy. The article starts with a brief overview of the literature and methodology before presenting, and then discussing, the findings.

\begin{tabular}{|ll}
\hline Glossary & \\
\hline Aiga & $\begin{array}{l}\text { extended family, can mean nuclear family, but in } \\
\text { this study it refers to a family that comprises several } \\
\text { generations }\end{array}$ \\
Fa'asamoa & $\begin{array}{l}\text { Māori name for New Zealand; Māori were the first } \\
\text { settlers of New Zealand }\end{array}$ \\
the Samoan way, which consists of language, customs, \\
culture and traditional norms
\end{tabular}

\section{LITERATURE REVIEW}

\section{Nutrition, food insecurity and obesity over the lifespan}

Experts agree that, throughout the life course, a diet of a combination of wholesome, relatively unprocessed foods, mainly plants, is best for health, immunity and longevity (Katz \& Meller, 2014). Optimal eating is of paramount importance at times of rapid development and growth, i.e., during pregnancy, childhood, and adolescence. Globally, and in Aotearoa NZ, food-based dietary guidelines (Food and Agriculture Organization of the United Nations [FAO], 2020; MOH, 2018) recommend a variety of foods and frequent movement, particularly among Māori and Pasefika populations (Borrows et al., 2011).

According to Stats NZ (2019), Pasefika people make up 8.1\% of the overall Aotearoa NZ population and they have a median age of 23 years. Pasefika people have the highest prevalence of food insecurity; $40 \%$ of Pasefika children live in food insecure households (MOH, 2019a) and 67\% of the Aotearoa NZ Pasefika population are classified as obese, with female obesity prevalence higher than male at 70\% and 63\% respectively ( $\mathrm{MOH}, 2019 \mathrm{~b})$. Food insecurity, mainly driven by a lack of money for food, is associated with poor nutrition and obesity in children and adults. Many authors have described food insecurity as a growing concern, affecting 7.3\% of households in Aotearoa NZ (Jackson \& Graham, 2017; MOH, 2012). Poor nutrition, obesity and food insecurity become particularly significant during pregnancy. In South Auckland, the high number of morbidly obese pregnant women has become the new normal (Counties Manukau District Health Board [CMDHB], 2013, 2019; Raven \& StewartWithers, 2019).

Based on their critical discourse analysis of 30 government, media and academic documents, Raven and Stewart-Withers (2019) argue that society regards pregnant women as being solely responsible for their own nutrition. They argue that, in fact, for some women malnutrition is more the result of food insecurity and disempowerment. They maintain that midwives need to - among other things - advocate for "woman-centred, policy-based approaches towards nutrition, whereby the underlying drivers of poverty are actively addressed" (Raven \& Stewart-Withers, 2019, p. 35).
They assert that "[i]f these issues are to be truly addressed then a more nuanced understanding of reasons for women's malnutrition during pregnancy is required" (Raven \& Stewart-Withers, 2019, p. 36).

Obesity is a form of malnutrition, with cheap foods not meeting the nutritional requirements for optimal health (Rush, 2009). Signal et al. (2013) believe the lack of food security is a "wicked" problem because it is complicated. There are many causes and no one solution. It is generally agreed that an environmental approach is needed to reduce food insecurity. This includes personal behaviours in the context of the physical, economic, sociocultural and political environments (Signal et al., 2013). However, Raven and Stewart-Withers (2019), also citing Pearce et al. (2011), argue that obesity "is strongly linked to an obesogenic environment, where culture, employment opportunities, the local housing market, and access to transport and healthy food impact on food accessibility and choices" (p. 37). People in the most economically deprived areas live in an obesogenic environment, with a higher than average representation of fast food outlets (Swinburn, 2020). Moreover, for a person being paid low wages, this makes the convenience of tasty but low-nutrient takeaway food all the more attractive. The Samoan women in this study made changes in what they ate and how much they moved, within the constraints of the environment, mentioning midwives as positive change influencers.

\section{Researcher positionality}

The lead author is a Samoan woman and working mother, who has experienced many of the constraints, including but not limited to: commitment to the extended family, church and wider Pasefika community, limited disposable income, time combining work and study, and juggling different work commitments. The lead author grew up in Samoa and completed her schooling there before moving to Aotearoa NZ. This has allowed her further insights into both life in Samoa and life in Aotearoa NZ with all the many challenges. It also gave her an in-depth familiarity with fa' asamoa and the ability to interact with the older generation in Samoan, in a respectful and culturally appropriate manner. This was very important in the study as culturally inappropriate communication may be a significant barrier to talanoa with older generations. The lead author watched her mother struggle with, and succumb to, the complications of Type 2 diabetes mellitus (T2DM) and this was the main impetus for her to undertake her study. The links between obesity, gestational diabetes mellitus (GDM) and a predisposition to developing the metabolic disease T2DM later in life have been well documented (Agarwal et al., 2018), as has the important role midwives play in providing guidance (Arrish et al., 2016).

The term "health literacy" emerged to describe knowledge and skills individuals need to make informed choices about their health (Vidgen \& Gallegos, 2014). The World Health Organization defines health literacy as "the cognitive and social skills which determine the ability of individuals to gain access to, understand and use information in ways which promote and maintain good health" (Nutbeam, 2008, p. 2074). Similarly, food literacy can be related to an individual's understanding of dietary intake and its influence on health (Velardo, 2015). Studies have shown the importance of food literacy (Gallegos \& Vidgen, 2010; Vidgen \& Gallegos, 2011); the environment, including financial, transport, housing and climate considerations (Cullen et al., 2015); and culture (Snyder, 2009). For Pasefika people, food is also about connectedness and relationships at individual, family and societal levels. Ahio (2011) found that, for Tongan and other Pasefika peoples, providing food might be more about quantity than quality. 
The question is whether current health messages are effective at reaching Pasefika people. Researchers such as Savila and Rush (2014) and Okesene-Gafa et al. (2016) emphasise the importance of maternal health and maternal lifestyle choices in relation to the health of their children.

Midwives aim to work in partnership with women (Krisjanous \& Maude, 2014). Working in partnership entails empowering women to make informed decisions about their own health and that of their unborn babies. Tui Atua (2009) describes the role of the traditional Samoan fa'atosaga, who is seen as the promotor and protector of human life. In their 2013 study, Garnweidner et al. (2013) found that midwives proved to be effective and trustworthy when they promoted a healthy diet and provided associated information to pregnant women, something that also resonates with findings by Wilhelmova et al. (2015). Szwajcer et al. (2009) argue that midwives can help pregnant women by addressing specific nutrition-related questions during consultation. Similarly, Boyle and colleagues (2016) assert that it is important to develop a partnership relationship between midwives and pregnant women, which will enable midwives to provide care and support in response to pregnant women's needs. There have been no previous reports in Aotearoa NZ about the role of midwives in the food literacy of Pasefika women, nor of the intergenerational transmission of knowledge.

The current paper reports on semi-structured interviews with 15 women from five Samoan families in the South Auckland area of Auckland, Aotearoa NZ, and includes their comments on what they learned from their midwives.

\section{AIM}

The aim of the original study (Wong Soon, 2016) was to firstly explore the extent of understanding of food literacy among three generations of women in Samoan families, all related along matrilineal lines. Secondly, the study aimed to examine how each of the three generations navigated the food system to ensure that their dietary intake contributed to the quality of their health and was consistent with nutritional recommendations. Lastly, the study aimed to identify whether food literacy was influenced within a generation (intragenerationally), or between and across the generations (intergenerationally), or both. The aim of this article is to report on the role of midwives as positive influencers on raising the level of women's food literacy.

\section{METHOD}

The study involved a modified Delphi method in combination with the talanoa approach.

The Delphi approach involves a staged process and uses a panel of experts (Fletcher \& Marchildon, 2014; Graham et al., 2003; Norcross et al., 2002; Rowe \& Wright, 2011). This study did not use the full traditional Delphi method but used elements from the method to find out the most consistent consensus, based on the views of participants who are the "experts" in the topic being examined. The initial round of the Delphi process (Round One) enabled the author to generate ideas and uncover the issues relating to the topic being examined. The researcher summarised the participants' first interviews and provided feedback to the participants who then reviewed and discussed their initial responses in Round Two until consensus was reached.

Tunufa $i$ (2016) traces the etymology and meaning of talanoa and its relevance to different Pasefika populations. Clery (2014) describes talanoa as to chat, to yarn, and to tell stories, writing: "[t]alanoa is context specific and responds to the needs of people in a given situation... It functions to strengthen relationships" (p. 108). For their study, Schleser and Firestone (2018) describe talanoa as an "appropriate Pacific research method... because it is based on a deep interpersonal relationship between the participants" (p. 165).

The lead author combined the Delphi and talanoa approaches because this allowed her to have multiple communications with each participant. The lead author particularly wanted to explore the role the extended aiga has on food choices.

This combined technique was appropriate to use in this study because of the focus on drawing views from the Samoan women as experts and what it meant for their health. All participants were related along the maternal side because the lead author wanted to see if there was any intergenerational influence affecting these women in terms of food literacy.

Two rounds of Delphi-Talanoa sessions were completed. The 15 semi-structured interviews in the first round ranged in length from 30 minutes to two hours. A summary of responses was reviewed and clarified in the second round.

\section{Recruitment and selection}

Participants were recruited, using the snowballing technique (Streeton et al., 2004), through churches, school alumni and village subcommittee networks in the South Auckland area of Auckland, Aotearoa NZ. While this is not a usual recruitment technique used with the Delphi method, the talanoa approach involves stakeholders and the women interviewed were stakeholders and experts because they were Samoan women involved in making food choices for their families.

\section{Analysis}

All interviews were transcribed by the lead author. NVivo 11 (Bazeley \& Jackson, 2013) was used to extract, code and recode the themes emerging from both rounds of talanoa. The transcribed interviews and coding were reviewed with the thesis supervisors and co-authors (IC, ER) for consistency and validity.

The authors used thematic analysis to analyse recurrent themes from the talanoa.

\section{Ethics}

Ethics approval was granted by the Auckland University of Technology Ethics Committee (AUTEC) under number 15/179.

\section{FINDINGS}

The 15 women participants were divided into three groups, in accordance with their status within the families. The oldest group are referred to as "the grandmothers" and ranged in age between 65 and 85 . Two of the five grandmothers had completed primary education; while two had completed secondary education (in Samoa) and one had completed tertiary level education in both Samoa and Aotearoa NZ. The second group of women participants are referred to as "the mothers". They ranged in age between 41 and 59; four had completed tertiary level education in Aotearoa NZ and one had completed secondary schooling in Samoa. The youngest participants are referred to as "the granddaughters" and ranged in age between 18 and 38. Three of the granddaughters had tertiary level education and two had completed secondary education.

The following final themes emerged from the two rounds of talanoa:

1. An understanding of food literacy

2. The role of midwives and other health professionals 
3. The importance of attending community programmes

4. Sharing information within the family environment

The focus of this paper is on themes two and four; the other themes have been published elsewhere (Wong Soon, 2016).

\section{The role of midwives in all five families}

\section{Family 1}

In Family 1, Mother 1 (M1) explained how midwives had encouraged her understanding of the importance of reading food labels for information about nutrients. Pointing at the nutritional label on a loaf of brown bread, she said:

\section{Before I didn't understand it. When the health professionals such as my midwives informed me, I started researching things like nutrients and what was on the back here.}

Within Family 1, M1 was the main informant who shared what she had learned in terms of health and food literacy with other women in the family. She said that her midwife had emphasised the importance of healthy eating during pregnancy. M1 said:

During my pregnancies, there was a lot of sessions with midwives and pregnant women about balanced meals and cooking food such as preparations and things that should not be mixed in food or you would get sick. That was the time I got more information. They also advised me to attend community health programmes that some health professionals provide services to help improve knowledge on a healthy lifestyle from those programmes.

Reading this [referring to brown bread nutritional information] and knowing what was good for me when I was pregnant. My midwives and family doctor showed and explained these, making me understand and I got to eat in moderation.

Based on M1's quote above, she shared this information with Grandmother 1 (GM1) and Granddaughter 1 (GD1) and other members of her aiga. M1 conveyed to the other two family members the benefits of eating healthy food and in moderation. Other health professionals mentioned included the family doctor, diabetes nurse and dietitian.

\section{Family 2}

In Family 2, it was the grandmother (GM2) who shared her knowledge of food literacy and healthy eating with other members of the aiga. GM2 had continued her practice of having a vegetable garden when she moved to Aotearoa NZ from Samoa. GM2 had also undertaken tertiary level training in nutrition at the University of the South Pacific and shared her knowledge with her family. She said:

\section{It is good because of different nutrients that are used in your body - those nutrients that we get from different foods such as iron, calcium, it is good for your eyes; you will also get different vitamins that are good for your bones. It is good [for] the blood and the body.}

GM2's daughter (Mother 2 [M2]), said that her midwife and family doctor had also provided her with important information about a nutritional dietary intake and a healthy lifestyle. The granddaughter (Granddaughter 2 [GD2]) specifically mentioned the advice she received from her midwife about maintaining a healthy diet and body weight, through eating a variety of nutritious foods, portion control and physical activity. GD2 said:

When I was pregnant ... I tried to eat good food for her [pointing at her 2-month old daughter] ... Eating heaps of vegetables, fish, chicken and stuff... In the right amount, because I don't want to overeat.

GD2's midwife had advised her that any choices GD2 made could affect her baby's health, and GD2 commented on this in the context of the life-changing experience of becoming a mother.

\section{Family 3}

In Family 3, the mother (Mother 3 [M3]) and the granddaughter (Granddaughter 3 [GD3]) acted as main sources of information for other family members. Again, different health professionals had played a role in encouraging the family's understanding of a healthy diet and lifestyle choices. GD3 stated that she had learned most from midwives as they had been more specific and "simple" in how they explained to her what she should do in order to have healthy pregnancies. She said the midwives were the ones who had recommended that she should take part in community health classes, the better to manage her GDM. GD3 said:
I know it is important to eat a balanced meal. A portion of meat, some vegetables. I feel it is important to know how much I am eating. If this is what I get on my plate, I eat it, and not go for a second serving, because you will get sick. This was the advice that I got from my midwives and family doctor during all my pregnancies, because I had diabetes during my pregnancies.

One midwife had told her that attending community health programmes would help her not only find out about GDM, diet and physical activity, but also about health checks, walking groups and other community programmes where she could improve her knowledge around health. GD3 had told her midwife that, since she was her grandmother's fulltime carer, she would ask her grandmother (GM3) to come along to these community programmes. Both the midwife and the family doctor told GD3 that there were in fact special community programmes for older people. Overall, she had found the midwife's advice extremely beneficial, because by coming along, GD3 had learned about healthy living for seniors.

\section{Family 4}

In Family 4 it was Mother 4 (M4) who was the main source of information for the rest of the family. M4 had been diagnosed with GDM and her midwives had encouraged her to make changes to her diet and remain physically active, in order to have healthy pregnancies. M4 said:

\section{My family doctor and midwives advised me not to overeat, but [eat] in the right portions, in order to have healthy pregnancies. From there on I knew that it was not [about] eating a lot to keep my babies strong during my pregnancies, which is what my mother always told me: to eat more to keep my unborn baby strong during pregnancy.}

M4 in turn encouraged her mother (Grandmother 4 [GM4]) to make healthy lifestyle changes and attend community health programmes for the sake of the health of the "grand- and greatgrandchildren". Granddaughter 4 (GD4) mentioned that several family members, including her grandmother (GM4), had been diagnosed with borderline hypertension and diabetes, and that this had been a real impetus for her to make healthy lifestyle changes. She said she had learned a lot from her mother (M4), about healthy eating. This in turn had motivated GD4 to learn more about nutrition and to pursue health studies at one of the tertiary institutes.

Family 5 
A health scare had also been the impetus for the women in Family 5 to make healthy lifestyle changes, after Grandmother 5 (GM5) had suffered a stroke. Her daughter (Mother 5 [M5]), was the main source of advice on a healthy lifestyle. M5 believed that making healthy changes in terms of food and fitness should start within the home and that it was her task as a mother to make sure such changes took place. She said:

\section{My mother does not know [referring to nutritional information and other food labels] and my children are young. It is my task as a mother to know this information in order to cook balanced meals for my family.}

M5 said her midwives had taught her how to read food labels during pregnancy and that her pregnancies had changed her "view on food" and her habits. She had been diagnosed with GDM during her first two pregnancies and her midwives had asked her to try and be more physically active and change her dietary intake. M5 said:

\section{My midwives advised me when I got pregnancy diabetes. That was when I decided I must practise a healthy lifestyle with my family... my doctors also advised me and I saw that it was a mother's responsibility to prepare healthy meals for her family. No mother would want to see her children getting sick.}

M5 in turn had taught her daughter about the importance of a healthy diet and played a role in her joining the gym. Granddaughter 5 (GD5) referred to this sharing of information when she said:

My mum always talks about healthy food and what's healthy for us.

\section{DISCUSSION}

Two things became very clear. Firstly, midwives were positive influencers within Samoan families; their influence related to improving an understanding of choices impacting on the health of the unborn child. Midwives talked about portion sizes and vegetables but also made women aware of the importance of staying active and of programmes in the community. Secondly, whatever women had learned from the midwives, they then shared within the family. The positive influence of midwives resonated long after women had given birth. Mothers shared with their mothers - the grandmothers in the study - and also with their daughters, as they grew up.

The original study did not set out to explore the role of midwives or other positive influencers, and participants were not asked why they had felt supported by midwives. This was probably due to the original nature of the research with its focus on food literacy, rather than positive influencers, that such questions were not explored more in-depth. Similarly, women did not share comments about the ethnicity or culture of the midwives; however, some did share that in general they enjoyed having health conversations in their home language (i.e., Samoan). This might mean that it would be good to encourage more Samoan - and other Pasefika - women to become midwives.

The authors would speculate that midwives working in partnership with, and empowering, women resulted in the former being viewed by the women as positive influencers. Women then took control of what they were able to implement, within the constraints of their own social, financial and other circumstances. What came out through many interviews was that midwives shared information with women. Previous studies have shown that positive influencers can only be positive influencers when they have the trust of the other person, and we can assume this was the case here.

\section{STRENGTHS AND LIMITATIONS}

Limitations of the research include the small number of participants. The snowballing method may have led to "like" participants referring the researcher to each other. The findings of this small study add weight to the likelihood of the important role midwives play in ensuring the health of Samoan mothers and their families. Midwives equipping women with important information about nutritious food, portion control and physical activity had consequences in "ever increasing circles" in all of the families involved in the research.

As the findings are not generalisable and the study did not focus on positive influencers, it would be useful to conduct research that specifically focuses on midwives' role in influencing women's food and other lifestyle choices, particularly amongst groups with reported low levels of health literacy and/or high incidence of GDM.

\section{CONCLUSION}

The important role of midwives in increasing women's knowledge of healthy lifestyle choices was relayed by all those participants who had received antenatal care in New Zealand: M1, M2, M4, M5, GD2 and GD3. These participants all agreed that midwives had been their first source of advice in helping them identify the type of food that would benefit both the mother and her unborn child. They received information from midwives related to reading nutrition labels to identify nutritious food stuffs, physical activity, portion control, free health checks, and participating in community health programmes. Previous studies (Arrish et al., 2017) found that guidelines for Australian midwives as to how to provide information on food choices during pregnancy may be vague, yet the present study suggests that midwives were very successful in imparting important dietary advice to the Samoan women interviewed. The women interviewed followed this advice and also shared it with other women in their aiga. It is interesting to note that while the obesogenic environment itself did not change, women started making changes. Women made changes because they believed these were important for their families.

It is important that midwives are aware of what works and what does not, and that guidelines around healthy conversations in pregnancy are developed, as was the case in New Zealand (GRAVIDA, 2013). The importance of food literacy among Samoan women in furthering better health outcomes for themselves and their families cannot be overstated.

Women involved in meal preparation ensured healthier food choices were put in place to benefit the entire family. This aligns with findings by Rosenkranz and Dzewaltowski (2008) and Barker (2015) that parents and families influence children's dietary habits and that the home food environment is an important factor of this. Women felt it was their role to share health-related information with other members of the wider aiga. Increased food literacy also enabled women to ask more pertinent questions of their family doctors, which is aligned with findings by Crezee and Roat (2019) about the similar effect of improving health literacy in low-literate families. There is no doubt in the authors' minds that this was helped by midwives working in partnership with women, and talking to them on an equal footing. Trust played an important role and this is again in line with findings by Crezee and Roat (2019). Women trusted midwives and this trust resulted in midwives being positive influencers on the level of these Samoan women's food literacy. 


\section{CONFLICT OF INTEREST DISCLOSURE}

The authors declare that there are no conflicts of interest.

\section{Key messages}

- Midwives played an important role in helping a group of pregnant Samoan women understand about healthy eating during pregnancy

- In the study, women shared what they had learned about healthy eating from their midwives with other female relatives

- Learnings taken from midwives included the importance of choosing healthy foods, portion control and physical activity

\section{REFERENCES}

Agarwal, P., Morriseau, T. S., Kereliuk, S. M., Doucette, C. A., Wicklow, B. A., \& Dolinsky, V. W. (2018). Maternal obesity, diabetes during pregnancy and epigenetic mechanisms that influence the developmental origins of cardiometabolic disease in the offspring. Critical Reviews in Clinical Laboratory Sciences, 55(2), 71-101. https:// doi.org/10.1080/10408363.2017.1422109

Ahio, L. L. (2011). Vaevae Manava: context and perception of food security for Tongan mothers and health workers [Master's thesis, Auckland University of Technology]. https://openrepository.aut.ac.New Zealand/ handle/10292/3487

Arrish, J., Yeatman, H., \& Williamson, M. (2016). Australian midwives and provision of nutrition education during pregnancy: A cross sectional survey of nutrition knowledge, attitudes, and confidence. Women and Birth, 29(5), 455-464. https://doi.org/10.1016/j.wombi.2016.03.001 Arrish, J., Yeatman, H., \& Williamson, M. (2017). Midwives' role in providing nutrition advice during pregnancy: Meeting the challenges? A qualitative study. Nursing Research and Practice, 2017, Article 7698510. https://doi.org/10.1155/2017/7698510

Barker, M. (2015). Developmental origins, behaviour change and the new public health. Journal of Developmental Origins of Health and Disease, 6(5), 428-433. https://doi.org/10.1017/S2040174415001312

Barreiro-Hurlé, J., Gracia, A., \& de-Magistris, T. (2010). Does nutrition information on food products lead to healthier food choices? Food Policy, 35(3), 221-229. https://doi.org/10.1016/j.foodpol.2009.12.006

Bazeley, P., \& Jackson, K. (Eds.). (2013). Qualitative data analysis with NVivo. SAGE.

Borrows, J., Williams, M., Schluter, P., Paterson, J., \& Langitoto Helu, S. (2011). Pacific Islands Families Study: The association of infant health risk indicators and acculturation of Pacific Island mothers living in New Zealand. Journal of Cross-Cultural Psychology, 42(5), 699-724. https:// doi.org/10.1177/0022022110362750

Boyle, S., Thomas, H., \& Brooks, F. (2016). Women's views on partnership working with midwives during pregnancy and childbirth. Midwifery, 32, 21-29. https://doi.org/10.1016/j.midw.2015.09.001

Clery, T. N. (2014). Extending the Talanoa; Weaving Pacific and Performative Methods for Peace Research in Contemporary Fiji. In P. Fairburn-Dunlop \& E. Coxon (Eds.), Talanoa: Building a Pasifika research culture (pp. 105-128). Dunmore.

Counties Manukau District Health Board. (2013). Antenatal management of obesity/morbid obesity.

Crezee, I. H. M., \& Roat, C. E. (2019). Bilingual patient navigator or healthcare interpreter: What's the difference and why does it matter? Cogent Medicine, 6(1), 1-15. https://doi.org/10.1080/233120 5X.2019.1582576

Cullen, T., Hatch, J., Martin, W., Higgins, J. W., \& Sheppard, R. (2015). Food Literacy: Definition and Framework for Action. Canadian Journal of Dietetic Practice and Research, 76(3), 140-145. https://doi. org/10.3148/cjdpr-2015-010
Fletcher, A. J., \& Marchildon, G. P. (2014). Using the Delphi Method for Qualitative, Participatory Action Research in health leadership. International Journal of Qualitative Methods, 13(1), 1-18. https://doi. org/10.1177/160940691401300101

Food and Agriculture Organization of the United Nations. (2020). Food-based dietary guidelines. http://www.fao.org/nutrition/nutritioneducation/food-dietary-guidelines/en/

Gallegos, D., \& Vidgen, H. A. (2010). Food literacy: time for a new term or just another buzzword? Journal of the Home Economics Institute of Australia, 17(2), 2-8.

Garnweidner, L. M., Pettersen, K. S., \& Mosdøl, A. (2013). Experiences with nutrition-related information during antenatal care of pregnant women of different ethnic backgrounds residing in the area of Oslo, Norway. Midwifery, 29(12), e130-e137. https://doi.org/10.1016/j. midw.2012.12.006

Graham, B., Regehr, G., \& Wright, J. G. (2003). Delphi as a method to establish consensus for diagnostic criteria. Journal of Clinical Epidemiology, 56(12), 1150-1156. https://doi.org/10.1016/S08954356(03)00211-7

GRAVIDA. (2013). Healthy Conversation Skills training. http:// www.gravida.org.New Zealand/what-we-do/translation-projects-andprogrammes/gravida-healthy-start-workforce/healthy-conversation-skillstraining/

Jackson, K., \& Graham, R. (2017). When dollar loaves are all you have: Experiences of food insecurity in Hamilton, New Zealand. In S. Groot, C. Van Ommen, B. Masters-Awatere, \& N. Tassell-Matamua (Eds.), Precarity. Uncertain, insecure and unequal lives in Aotearoa New Zealand (pp. 63-75). Massey University Press.

Katz, D. L., \& Meller, S. (2014). Can we say what diet is best for health? Annual Review of Public Health, 35(1), 83-103. https://doi.org/10.1146/ annurev-publhealth-032013-182351

Krisjanous, J., \& Maude, R. (2014). Customer value co-creation within partnership models of health care: An examination of the New Zealand Midwifery Partnership Model. Australasian Marketing Journal, 22(3), 230-237. https://doi.org/10.1016/j.ausmj.2014.08.005

Machín, L., Giménez, A., Curutchet, M. R., Martínez, J., \& Ares, G. (2016). Motives underlying food choice for children and perception of nutritional information among low-income mothers in a Latin American country. Journal of Nutrition Education and Behaviour, 48(7), 478-485. e471 https://doi.org/10.1016/j.jneb.2016.04.396

Ministry of Health. (2012). A Focus on Pacific Nutrition: Findings from the 2008/09 New Zealand Adult Nutrition Survey. https://www.health. govt.New Zealand/publication/focus-nutrition-key-findings-2008-09New Zealand-adult-nutrition-survey

Ministry of Health. (2018). Eating and activity guidelines: Guidelines statements for New Zealand adults. https://www.health.govt.New Zealand/our-work/eating-and-activity-guidelines

Ministry of Health. (2019a). Household food insecurity among children in New Zealand. https://www.health.govt.New Zealand/system/files/ documents/publications/household-food-insecurity-among-childrennew-zealand-health-survey-jun19.pdf

Ministry of Health. (2019b). New Zealand Health Survey: Annual data explorer. https://minhealthNew Zealand.shinyapps.io/New Zealandhealth-survey-2018-19-annual-data-explorer

Norcross, J. C., Hedges, M., \& Prochaska, J. O. (2002). The face of 2010: A Delphi poll on the future of psychotherapy. Professional Psychology: Research and Practice, 33(3), 316-322. https://doi. org/10.1037/0735-7028.33.3.316

Nutbeam, D. (2008). The evolving concept of health literacy. Social Science \& Medicine, 67(12), 2072-2078. https://doi.org/10.1016/j. socscimed.2008.09.050

Okesene-Gafa, K., Chelimo, C., Chua, S., Henning, M., \& McCowan, L. (2016). Knowledge and beliefs about nutrition and physical activity during pregnancy in women from South Auckland region, New Zealand. Australian and New Zealand Journal of Obstetrics and Gynaecology, 56(5), 471-483. https://doi.org/10.1111/ajo.12456

Pearce, J. R., Richardson, E. A., Mitchell, R. J., \& Shortt, N. K. (2011). Environmental justice and health: A study of multiple environmental deprivation and geographical inequalities in health in New Zealand. Social Science o Medicine, 73(3), 410-420. https://doi.org/10.1016/j. socscimed.2011.05.039

Raven, B., \& Stewart-Withers, R. (2019). Using critical discourse analysis and the concept of food security to understand pregnant women's nutrition in Aotearoa/New Zealand. New Zealand College of Midwives Journal, (55)35-43. https://doi.org/10.12784/nzcomjn155.2019.5.35-43 
Rosenkranz, R. R., \& Dzewaltowski, D. A. (2008). Model of the home food environment pertaining to childhood obesity. Nutrition Reviews, 66(3), 123-140. https://doi.org/10.1111/j.1753-4887.2008.00017.x

Rowe, G., \& Wright, G. (2011). The Delphi technique: Past, present, and future prospects-Introduction to the special issue. Technological Forecasting and Social Change, 78(9), 1487-1490. https://doi. org/10.1016/j.techfore.2011.09.002

Rush, E. C. (2009). Food Security for Pacific Peoples in New Zealand. A report for the Obesity Action Coalition. https://ana.org. New Zealand/wpcontent/uploads/2016/11/PacificfoodsecurityreportfinalMarch09_3.pdf

Savila, F., \& Rush, E. (2014). Pathways to health and wellbeing for Pacific children - how are we tracking? Pathways, 127(1404), 67-70. https://www. New Zealandma.org.New Zealand/journal-articles/pathways-to-healthand-wellbeing-for-pacific-children-how-are-we-tracking

Schleser, M., \& Firestone, R. (2018). Pasifika Youth and Health Perspectives: Creative transformation through smartphone filmmaking and digital Talanoa. In M. Schleser \& M. Berry (Eds.), Mobile story making in an age of smartphones (pp. 161-171). Springer. https://doi. org/10.1007/978-3-319-76795-6_16

Signal, L. N., Walton, M. D., Ni Mhurchu, C., Maddison, R., Bowers, S. G., Carter, K. N., Gorton, D., Heta, C., Lanumata, T. S., McKerchar, C. W., O'Dea, D., \& Pearce, J. (2013). Tackling 'wicked' health promotion problems: A New Zealand case study. Health Promotion International, 28(1), 84-94. https://doi.org/10.1093/heapro/das006 Snyder, S. (2009). Ethnographies of taste: Cooking, cuisine, and cultural literacy. Journal of Agricultural and Environmental Ethics, 22(3), 273283. https://doi.org/10.1007/s10806-009-9149-6

Stats NZ. (2019). Census population and dwelling counts. https://www. stats.govt.New Zealand/information-releases/2018-census-populationand-dwelling-counts

Streeton, R., Cooke, M., \& Campbell, J. (2004). Researching the researchers: Using a snowballing technique. Nurse Researcher, 12(1), 3547. https://doi.org/10.7748/nr2004.07.12.1.35.c5929

Swinburn, B. (2020, October 11). Sunday - Fat Shame. TVNew Zealand. https://www.tvNew Zealand.co.New Zealand/shows/sunday/clips/fatshame

Szwajcer, E. M., Hiddink, G. J., Koelen, M. A., \& van Woerkum, C. M. (2009). Written nutrition communication in midwifery practice: What purpose does it serve? Midwifery, 25(5), 509-517. https://doi. org/10.1016/j.midw.2007.10.005

Tui Atua, T. T. T. i. E. (2009). Bioethics and the Samoan indigenous reference. International Social Science Journal, 60(195), 115-124. https:// doi.org/10.1111/j.1468-2451.2009.01705.x

Tunufa'i, L. (2016). Pacific research: Rethinking the

Talanoa'methodology'. New Zealand Sociology, 31(7), 227-239. https:// search.informit.com.au/documentSummary; dn=561428501206421;res= IELNew ZealandC

Velardo, S. (2015). The Nuances of Health Literacy, Nutrition Literacy, and Food Literacy. Journal of Nutrition Education and Behavior, 47(4), 385-389.e381. https://doi.org/10.1016/j.jneb.2015.04.328

Vidgen, H. A., \& Gallegos, D. (2011). What is food literacy and does it influence what we eat: A study of Australian food experts. Queensland University of Technology.

Vidgen, H. A., \& Gallegos, D. (2014). Defining food literacy and its components. Appetite, 76(50-59). https://doi.org/10.1016/j. appet.2014.01.010

Wilhelmova, R., Hruba, D., \& Vesela, L. (2015). Key Determinants Influencing the Health Literacy of Pregnant Women in the Czech Republic. Slovenian Journal of Public Health, 54(1), 27-36. https://doi. org/10.1515/sjph-2015-0004

Wong Soon, H. N. (2016). Food Literacy: What does Food Literacy mean for Samoan families? [Master's thesis, Auckland University of Technology]. https://openrepository.aut.ac.New Zealand/ handle/10292/10497

\section{Accepted for Publication November 2020}

Wong Soon, H. N., Crezee, I., \& Rush, E. (2021). The role of New Zealand midwives as positive influencers on food literacy with Samoan families: Report on a small Auckland-based study. New Zealand College of Midwives Journal, 57, 5-11.

https://doi.org/10.12784/nzcomjnl57.2021.1.5-11 